\title{
Noise Suppression of Incoherent Light Using a Gain-Saturated SOA: Implications for Spectrum-Sliced WDM Systems
}

\author{
Anoma D. McCoy, Member, IEEE, Member, OSA, Peter Horak, Benn C. Thomsen, \\ Morten Ibsen, Member, OSA, and David J. Richardson, Fellow, OSA
}

\begin{abstract}
In this paper, we present an experimental and numerical study of semiconductor optical amplifier (SOA)-based noise suppression and its relevance to high-channel-density spectrumsliced wavelength-division-multiplexed systems. We show that the improvement in signal quality is accompanied by spectral distortion, which renders it susceptible to deterioration in the presence of subsequent optical filtering. This phenomenon originates from the loss of intensity correlation between spectral components of the SOA output when the signal spectrum is altered. As a consequence, a design tradeoff is introduced between intensity noise and crosstalk in high-channel-density systems. These adverse effects can be overcome by optimized SOA design, resulting in a significant improvement in signal quality.
\end{abstract}

Index Terms-Optical noise, semiconductor optical amplifier (SOA), spectrum slicing, wavelength-division multiplexing(WDM).

\section{INTRODUCTION}

$\mathbf{P}$ ASSIVE optical networks (PONs) have emerged as one of the leading contenders in the race to meet the demand for low-cost, high-bandwidth metropolitan and access services [1]. Spectrum slicing is an attractive and viable technology for such PON architectures [2] as it capitalizes on the economical advantages of incoherent sources such as light-emitting diodes, superluminescent diodes, and fiber-based amplified spontaneous emission (ASE) sources. Spectrum slicing is a method of sharing a single broadband optical source among many user channels by allocating a unique spectral slice to each channel [3]. However, the excess intensity noise present in these "thermal-like" incoherent sources imposes an upper limit on the achievable signal-to-noise ratio (SNR). System performance can only be improved by decreasing the bit rate or increasing the channel bandwidth, thus sacrificing system capacity [4].

To address this limitation of incoherent sources, a number of intensity-noise suppression techniques have been investigated. An optoelectronic compensation scheme was proposed in [5], where the noise was reduced by subtracting out the fluctuations

Manuscript received November 1, 2004; revised May 3, 2005.

A. D. McCoy, P. Horak, M. Ibsen, and D. J. Richardson are with the Optoelectronics Research Centre, University of Southampton, Southampton SO17 1BJ, U.K

B. C. Thomsen was with the Optoelectronics Research Centre, University of Southampton, U.K. He is now with the Department of Electronic and Electrical Engineering, University College London, London, WC1E 7JE, U.K.

Digital Object Identifier 10.1109/JLT.2005.852023 from the forward propagating light. However, the precise phase and gain matching that is required in the associated radiofrequency circuitry results in increased system complexity. An all-optical method using intrachannel four-wave mixing (FWM) has also been demonstrated to reduce intensity noise by significantly increasing the received channel bandwidth, and thus the SNR [6]. The FWM was produced at the receiver using a high-power erbium-doped fiber amplifier (EDFA) and $20 \mathrm{~km}$ of dispersion-shifted fiber.

Another all-optical technique uses the nonlinearities of a saturated semiconductor optical amplifier (SOA) to improve the signal quality of the transmitted light [7], [8]. This technique is more compact than the bandwidth expansion using dispersionshifted fiber and offers additional benefits in that the SOA can be used simultaneously for both signal modulation and amplification [9], [10]. The SOA efficiently suppresses the intensity fluctuations in the input light across a bandwidth determined by the device operating point and carrier lifetime, and can be used with channel bit rates as high as several gigabits per second. Reducing the source intensity noise enables increased channel granularity and spectral efficiency.

However, optical filtering after the SOA [e.g., in a wavelength division demultiplexer] results in increased intensity noise, thus reducing the noise-suppression benefit and degrading the received signal quality [11]. We have shown that this effect is more pronounced for narrow, steep input spectrum slices, where the nonlinearities of the SOA cause significant spectral distortion and broadening. In this paper, we present a comprehensive study of SOA-based noise reduction and its application to high-channel-density spectrum-sliced wavelengthdivision-multiplexing (WDM) systems.

The paper is organized as follows. In Section II, we describe the general characteristics and benefits of SOA-based noise reduction with emphasis on the achievable suppression bandwidth. Section III focuses on the observed spectral distortion of the intensity-smoothed SOA output and the signal degradation resulting from onward filtering. Section IV is then devoted to understanding the physical origins of the observed signal degradation. System issues associated with this approach are addressed in Section V, and in Section VI we discuss potential techniques to overcome the filtering effects and improve system performance. The added benefit of the saturated SOA for simultaneous noise suppression and modulation is discussed in Section VII. 


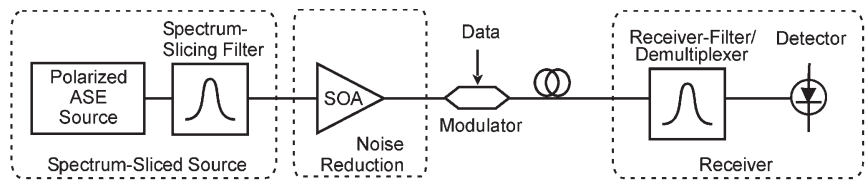

Fig. 1. Block diagram of single-channel spectrum-sliced system incorporating a saturated SOA for intensity-noise reduction.
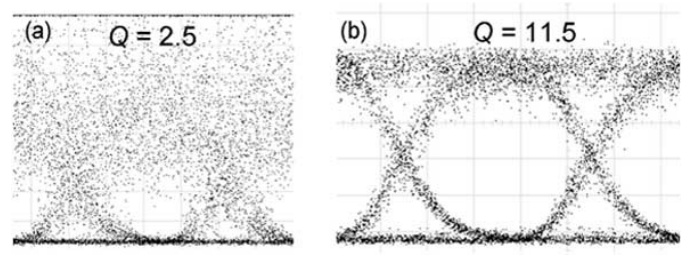

Fig. 2. Eye quality (a) before and (b) after SOA-based noise reduction for a $0.24-\mathrm{nm}$ input spectrum slice at a bit rate of $2.5 \mathrm{~Gb} / \mathrm{s}$.

\section{Characteristics OF THE NOISE-SUPPRESSION PROCESS}

Excess photon noise present in thermal-like incoherent light [12] imposes an upper limit on the achievable system performance in techniques employing incoherent sources. The noise power scales with the square of the detected optical intensity and, thus, the SNR cannot be improved by increasing the signal power [13]. The noise also depends on the shape and width of the input spectrum slice; it decreases with increasing spectral width [14]. Studies have shown that this intensity noise can be significantly reduced by introducing a saturated SOA at the transmitter before modulation [8], [15] (block diagram shown in Fig. 1). A representative example of the achievable performance improvement is presented in Fig. 2, which shows eye diagrams with and without noise reduction at a bit rate of $2.5 \mathrm{~Gb} / \mathrm{s}$. The simplicity and effectiveness of this technique makes it an attractive option for intensity-noise suppression of incoherent light.

To obtain maximum benefit, it is essential to understand the characteristics of the noise-suppression process. In this section, we discuss factors that control the noise mitigation offered by the SOA and examine their influence on the suppression bandwidth. The characterizations presented are based on the following experimental setup and procedure.

A polarized ASE source is spectrally sliced using a $0.24-\mathrm{nm}$ (30 GHz) 3-dB-bandwidth fiber Bragg grating (FBG). (Note that all filters used in this paper are specified in terms of their 3-dB bandwidth.) An EDFA is inserted after the FBG in order to ensure sufficient input power to saturate the SOA. The EDFA can be treated as being part of the ASE source and does not alter the thermal-like statistics of the light (as seen in the following discussions). The input power and polarization to the SOA are then optimized using a variable attenuator and polarization controller, respectively. The noise is characterized in terms of relative intensity noise (RIN), a figure of merit that directly quantifies the level of intensity noise of the continuous-wave (CW) signal. Single-frequency RIN measurements were performed at $100 \mathrm{MHz}$, using a 125-MHz low-noise photodetector and an electrical spectrum analyzer. RIN spectra were measured using a 22-GHz-bandwidth Agilent lightwave analyzer.

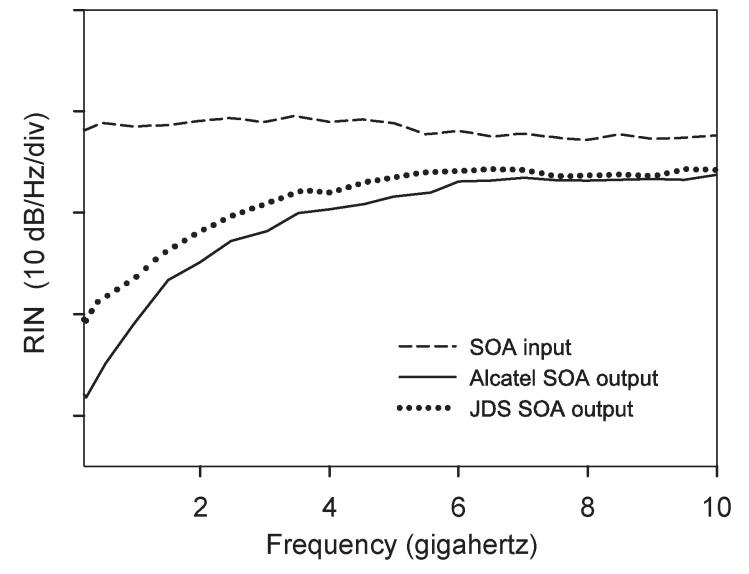

Fig. 3. RIN spectra for two different commercial SOAs. Input slice bandwidth is $0.24 \mathrm{~nm}$.

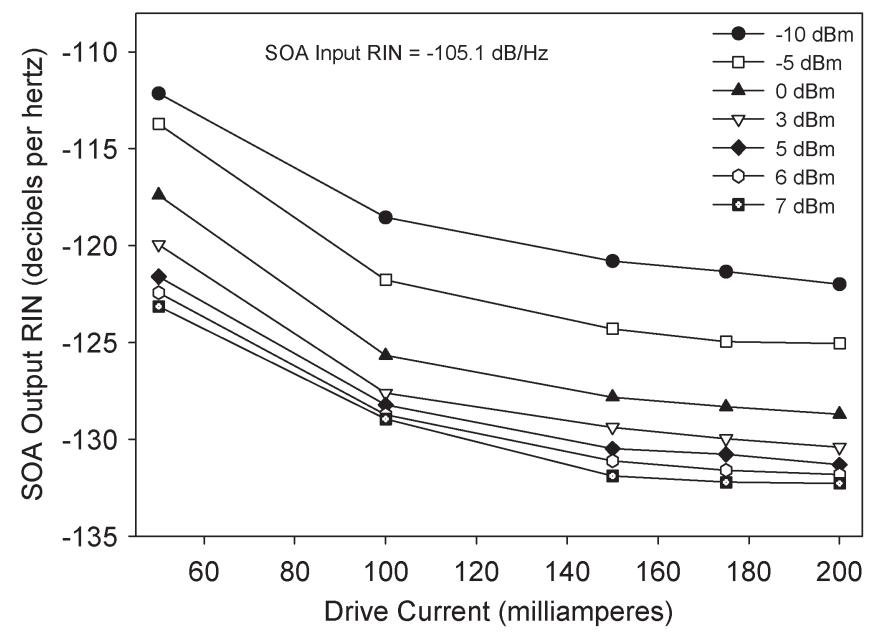

Fig. 4. SOA output RIN as a function of the amplifier drive current and input power. SOA input RIN for the $0.24-\mathrm{nm}$ spectrum slice is $-105.1 \mathrm{~dB} / \mathrm{Hz}$.

We first consider the noise power spectral density (PSD) before and after noise suppression for two different SOAs, over a $125-\mathrm{kHz}$ to $10-\mathrm{GHz}$ range (Fig. 3). The amplifiers are conventional bulk devices from Alcatel and JDS Uniphase, respectively. Although the SOAs are operated at approximately the same level of gain, the Alcatel SOA gives $\sim 7 \mathrm{~dB}$ greater suppression at low frequencies. This measurement qualitatively illustrates the dependence of the noise-suppression process on SOA device parameters.

We focus now on the noise suppression offered by a single SOA; unless otherwise specified, results presented in this paper are for the Alcatel SOA. As discussed in [15], the extent of the achievable noise suppression is influenced by the operating point of the amplifier. To ensure optimum performance for our experiments, this dependence is measured as a function of amplifier drive current and input power. We see from Fig. 4 that the RIN suppression does not improve appreciably for drive currents and input-power levels greater than $150 \mathrm{~mA}$ and $+5 \mathrm{dBm}$, respectively. However, high input power reduces the amplification benefit of the SOA, potentially resulting in increased cost and system complexity.

The suppression bandwidth also depends on the SOA device parameters and operating point. As discussed in [16], the 

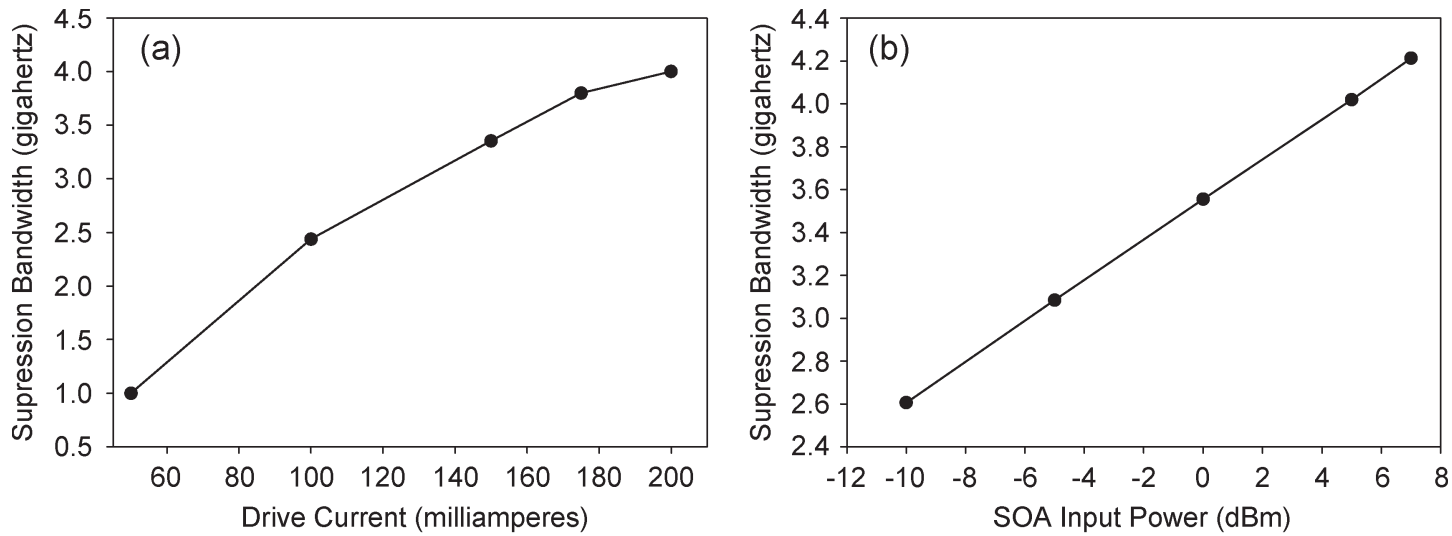

Fig. 5. Noise-suppression bandwidth as a function of (a) the drive current while the input power is fixed at $+5 \mathrm{dBm}$ and (b) the input power while the drive current is fixed at $200 \mathrm{~mA}$.

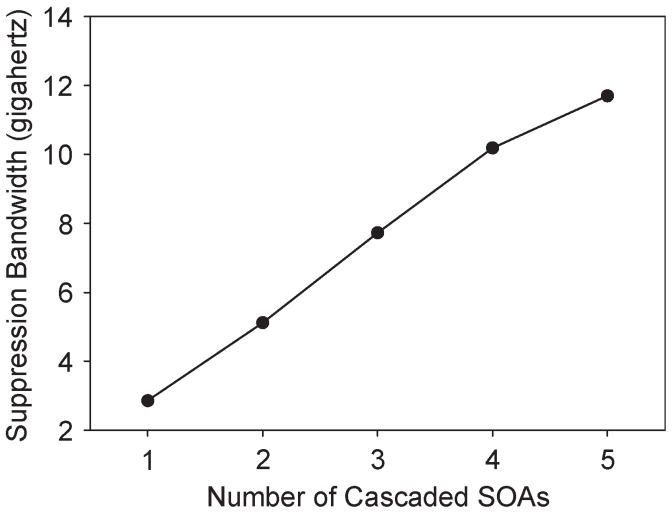

Fig. 6. Noise-suppression bandwidth as a function of the number of cascaded SOAs.

nonlinear gain dynamics of the SOA produce a dip in the noise PSD near dc, which is clearly visible in Fig. 3. This is in contrast with thermal-like light, which has an essentially white noise spectrum. The achievable suppression bandwidth directly determines the maximum bit rate and is therefore a critical parameter in any noise-compensation technique. For the purpose of these characterizations, the noise-suppression bandwidth is defined as the frequency at the half-power point from the maximum value of the noise-spectrum curve within the $10-\mathrm{GHz}$ bandwidth.

We investigated the dependence of the noise-suppression bandwidth on the device operating point by measuring the SOA output RIN spectra as a function of input power and drive current. In the first instance, we vary the SOA drive current while the input power is held constant at $+5 \mathrm{dBm}$. In the second characterization, we vary the SOA input power while the drive current is fixed at $200 \mathrm{~mA}$. The 3-dB suppression bandwidth is then determined for each measurement and is shown in Fig. 5. In Fig. 5(a), we see that as the drive current was varied from 50 to $200 \mathrm{~mA}$, the bandwidth improved from $\sim 1$ to $\sim 4 \mathrm{GHz}$. At fixed drive current [Fig. 5(b)], the bandwidth increased from $\sim 2.6$ to $\sim 4.2 \mathrm{GHz}$ as the input power was varied from -10 to $+7 \mathrm{dBm}$. It is clear from these results that the SOA offers appreciable suppression, even at relatively low saturation levels.

The noise-suppression bandwidth can also be improved by cascading SOAs in series as discussed in [17], and we examined
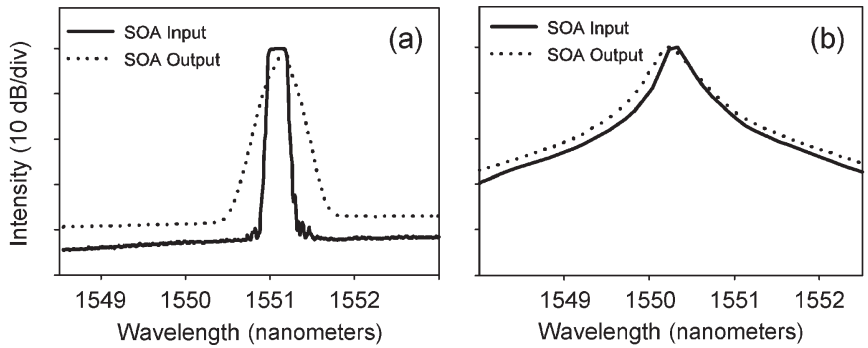

Fig. 7. Spectral broadening of the SOA output for two filter shapes, both with $\sim 0.24-$ nm bandwidths.

the effectiveness of this approach using a five-stage SOA arrangement. All five JDS amplifiers used in this experiment were operated at the same gain-compression level $(+5 \mathrm{dBm}$ input power, $450 \mathrm{~mA}$ drive current) and the SOA output RIN spectrum was measured for each additional amplifier stage. Fig. 6 shows the suppression bandwidth as a function of the number of SOAs. With a five-stage SOA cascade, we are able to achieve a suppression bandwidth of $12 \mathrm{GHz}$, a factor of four improvement over the single stage JDS SOA bandwidth of $\sim 3 \mathrm{GHz}$. These results are not directly comparable with a single-stage SOA with the same total device length, due to the additional attenuators and polarization controllers between each amplifier. The added ASE would also be reduced in the single-amplifier arrangement. However as seen from our results, conventional single-stage SOAs still offer substantial noise mitigation for bandwidths relevant to metro and access applications.

\section{Spectral Distortion And Post-SOA Filtering}

From a spectrum slicing point of view, the gain-saturated SOA offers an elegant solution to increase the spectral efficiency of the intensity-noise-limited system. However, closely spaced narrow transmitter and receiver filters are required to realize high channel counts. Our experiments have shown that spectral broadening introduced by the SOA is very pronounced for narrow-input spectral slices with steep roll-off. We illustrate this in Fig. 7, which shows the SOA spectral response to two filters of approximately the same $3-\mathrm{dB}$ bandwidth $(\sim 0.24 \mathrm{~nm})$, but with differing spectral shapes. It is clear that the steep 

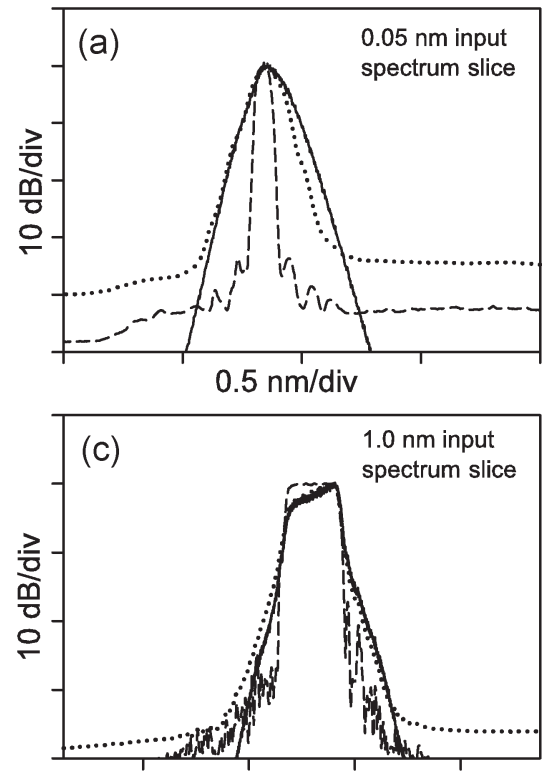

$2 \mathrm{~nm} / \mathrm{div}$
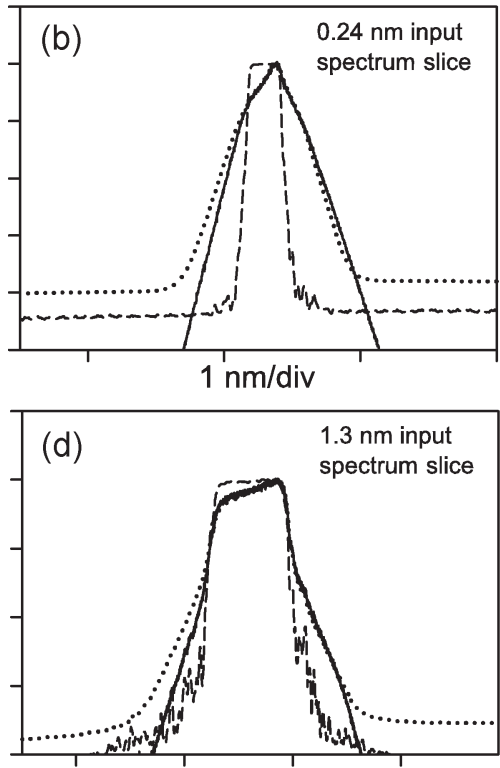

$2 \mathrm{~nm} / \mathrm{div}$

--- SOA Input $\quad$...... SOA output (expt)

- SOA output (sim)

Fig. 8. Spectra before and after the saturated SOA for 0.05-, 0.24-, 1.0-, and 1.3-nm spectrum slicing (FBG filters).

spectrum slice in Fig. 7(a), which has higher intensity noise, incurs significant spectral distortion. We have further shown that filtering this broadened output degrades the signal quality [11], counteracting the benefit offered by the saturated amplifier.

A numerical model was developed to better understand the observed spectral distortion and filtering effects; the nonlinear behavior of the saturated SOA was modeled using the field $E(z, t)$ and carrier density $N(z, t)$ equations [18]:

$$
\begin{aligned}
\frac{\partial E}{\partial z}+\frac{1}{v_{\mathrm{g}}} \frac{\partial E}{\partial t} & =\frac{\Gamma}{2}(1-i \alpha) g(N) E-\frac{\alpha_{\mathrm{int}}}{2} E \\
\frac{\partial N}{\partial t} & =\frac{I}{q V}-\frac{N}{\tau}-\frac{\Gamma}{\hbar \omega_{o}} g(N)|E|^{2} .
\end{aligned}
$$

Here, $v_{\mathrm{g}}$ is the group velocity, $\Gamma$ the mode confinement, $\alpha$ the linewidth-enhancement factor, $g(N)$ the gain coefficient, $\alpha_{\text {int }}$ the internal loss, $I$ the injected current, $q$ the electronic charge, $V$ the active layer volume of the device, $\tau$ the spontaneous carrier lifetime, and $\omega_{o}$ the input center frequency. Although the noise reduction can be modeled by the intensity and carrierdensity rate equations alone [15], the field equation is required to predict the spectral properties of the SOA output [19], including the effects of post-SOA filtering.

In our simulations, the spectrum-sliced input field is modeled using thermal light statistics. The amplifier is divided into short segments, where the length of each segment is small relative to the spatial variations of the input intensity. The rate equations are solved on this spatial grid using an ordinary differentialequation solver that calculates the field at the device output, $E(t, z=L)$.

We first consider the spectral-broadening effects introduced by the SOA for varying input bandwidths. As seen in Fig. 8, the numerical model accurately predicts the output spectra, clearly showing the broadening and distortion observed in the experimental measurements. Note that the ASE floor added by the SOA (less than $\sim 35 \mathrm{~dB}$ down from the main lobe) is not incorporated into the model. Good agreement between simulation and experiment indicate that this approximation is valid in most cases for the high signal strengths considered in this study. Some consequences of ignoring ASE are discussed in Section IV, and also in [20] and [21].

The broadening at the SOA output is caused by phasemodulation-induced chirp [18] and intrachannel FWM. The relative efficiencies of the two processes depend on the input slice bandwidth; wider slices generate more FWM products, while the chirp is more pronounced in the narrower spectral slices where the larger intensity fluctuations produce greater phase noise [19]. The phase modulation also has the effect of shifting the peak power toward longer wavelengths as the light travels through the amplifier [18]. This red shift is clearly visible in Fig. 8.

As stated previously, spectral filtering of this intensity smoothed output results in increased intensity noise, and thus reduced system performance. This is illustrated in Fig. 9 using single-channel (Fig. 1) $Q$ measurements at $2.5 \mathrm{~Gb} / \mathrm{s}$. A $\mathrm{LiNbO}_{3}$ electrooptic modulator (EOM) was used in these measurements, and $Q$ measurements were performed using a sampling oscilloscope with a $2.5-\mathrm{Gb} / \mathrm{s}$ optimized receiver module. Three different receiver filters of bandwidths 1.3, 0.7 , and $0.24 \mathrm{~nm}$ (same shape and bandwidth as input slice) were used for this experiment. The system performance with no SOA-based noise reduction is also shown for comparison. The corresponding spectra at the detector input are given in Fig. 10. The intensity-noise floor of the unfiltered SOA output occurs at $Q \approx 11.5$. However, using a steep 1.3 -nm receiver filter increased this floor to $Q \approx 10.2$. Although the signal degradation is noticeable here, the absolute measurement is low 


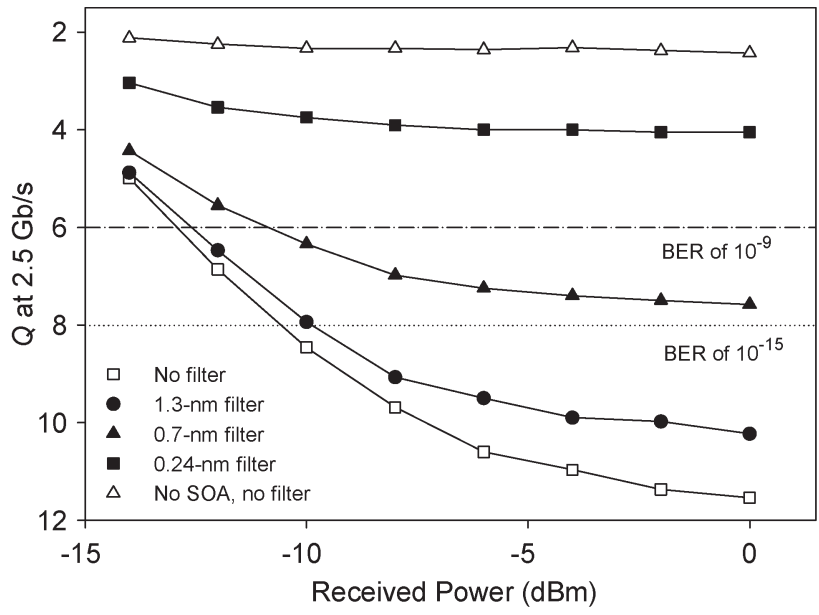

Fig. 9. Single-channel $Q$ measurements at $2.5 \mathrm{~Gb} / \mathrm{s}$ as a function of power and receiver filtering. $Q$ decreases with decreasing filter bandwidth. The input spectral slice is the 0.24-nm filter shown in Fig. 7(a).

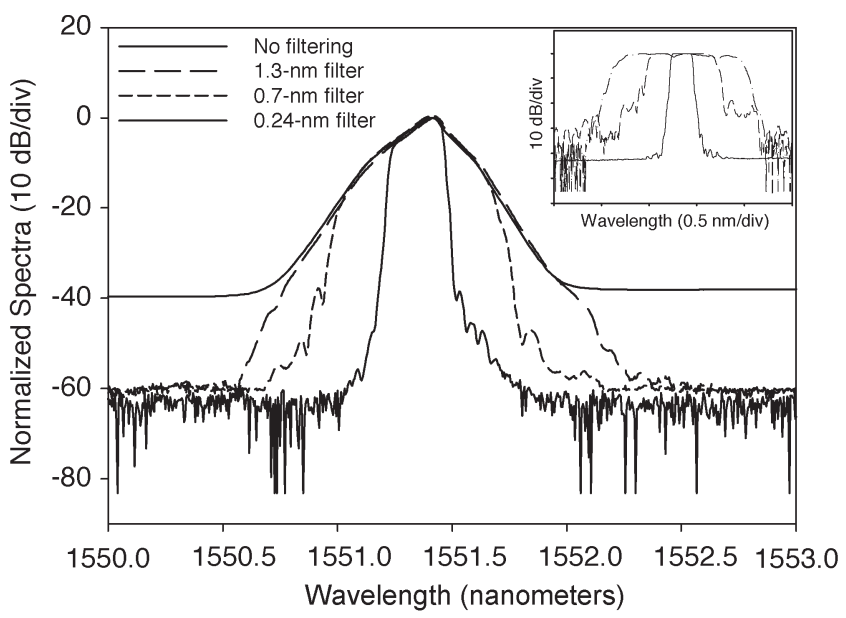

Fig. 10. Detector input spectrum (system output) for single channel only. Inset shows the receiver-filter transfer functions.

enough to be unnoticed in a routine bit-error-rate measurement. However, the narrower receiver filters $(0.7$ and $0.24 \mathrm{~nm})$ introduce significant system penalty.

We also measured the RIN of the post-SOA-filtered signal as a function of receiver filter bandwidth (Fig. 11), obtaining excellent agreement between experimental and simulation results. The figure also shows the SOA output RIN for the equivalent thermal-light spectrum (squares), calculated numerically using [14],

$$
\mathrm{RIN}=A \int_{-\infty}^{\infty} \psi\left(x+\frac{f}{2}\right) \psi\left(x-\frac{f}{2}\right) \mathrm{d} x .
$$

Here, $\psi(x)$ is the optical PSD, $f$ is the electrical frequency (100 MHz was used for our measurements), and $A$ is a constant that takes into account the optical-power-to-current conversion factor of the detector. Using (3), we are able to predict the RIN for an arbitrarily shaped incoherent light spectrum. The results show that, unlike thermal light, the intensity-smoothed output exhibits a pronounced increase in noise as the filter

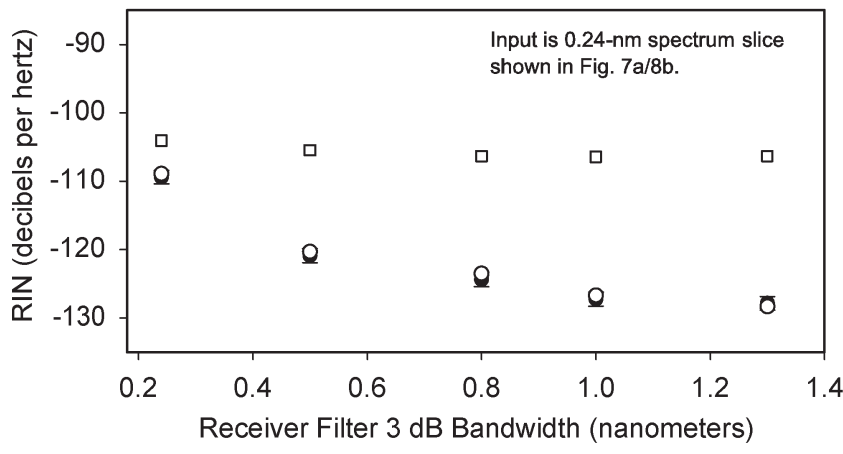

Fig. 11. Measured (•) and predicted (०) SOA output RIN for varying receiver filters. Simulation results include detector floor. SOA output RIN predicted from received spectra, using a thermal-light model, is also shown ( $\square)$. Receiver-filter spectra are as shown in Fig. 10 inset.

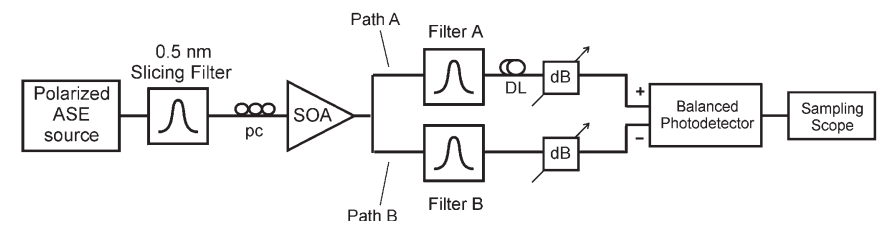

Fig. 12. Experimental setup to investigate the intensity correlation between different spectral components in the SOA input and output light. pc: Polarization controller. DL: Delay line.

bandwidth is reduced, suggesting a deviation from the statistics of incoherent light. This observed noise increase relates directly to the extent of the spectral filtering.

\section{ORIGIN OF POST-SOA FILTERING EFFECTS}

To understand the origins of the noise increase, it is important to appreciate the physical mechanisms underpinning the noisesuppression process. As suggested in [22], the nonlinearities that occur within the gain-saturated SOA cause interactions between the various spectral components present in the amplifier; this includes the original input signal, new frequencies generated by the nonlinear processes, and added ASE. We investigate the impact of these interactions on the statistical properties of the intensity-smoothed light by measuring the correlation between the intensity fluctuations of the different spectral components in the SOA output. Our experimental procedure is as follows.

Spectrum-sliced light of bandwidth $0.5 \mathrm{~nm}$ was launched into the saturated amplifier, and after amplification and intensity smoothing, the signal was split into two paths as shown in Fig. 12. Both paths contain 0.24-nm filters (filters A and B) that were used to slice the SOA output spectrum. The center wavelength of filter A was held fixed, while filter B was tuned across the SOA output spectrum; this is illustrated in Fig. 13. A delay line was used to match the path lengths of the two branches, and the powers of paths A and B were balanced for the zerooffset position of filter $\mathrm{B}$. The signals were then detected by an $800-\mathrm{MHz}$ balanced differential receiver and viewed on a highspeed sampling oscilloscope. The histogram function of the scope was used to measure the standard deviation of the fluctuations in the intensity difference between the two signal paths, as well as the standard deviation of the intensity noise in each 


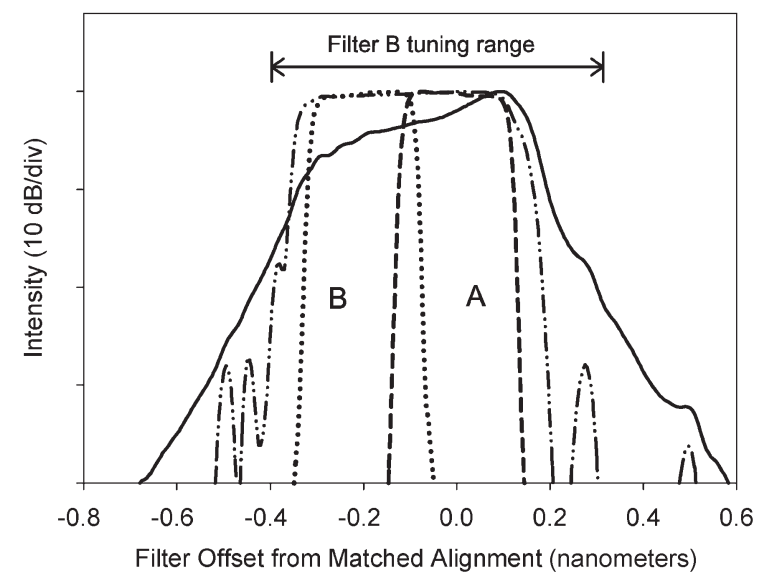

Fig. 13. Spectra of input slice (dash-dot line), SOA output (solid line), filter A (short dashes), and filter B (dotted line) for an offset of $-0.2 \mathrm{~nm}$.

path individually (using the shutters of the attenuators). These measurements were repeated while the center wavelength of filter B was scanned across the SOA output bandwidth.

The covariance of the intensity in the two frequency segments is then calculated using the well-known relation $\operatorname{var}(A-$ $B)=\operatorname{var}(A)+\operatorname{var}(B)-2 \operatorname{cov}(A, B)$ [23], where $\operatorname{var}(A)$ and $\operatorname{var}(B)$ represent the intensity variance of the signals in paths $\mathrm{A}$ and $\mathrm{B}$, respectively, and $\operatorname{cov}(A, B)$ is the covariance of the intensity in the two frequency segments. The correlation coefficient

$$
\rho=\frac{\operatorname{cov}(A, B)}{\sqrt{\operatorname{var}(A) \operatorname{var}(B)}}
$$

is then calculated, to compensate for the change in power as filter B is tuned across the SOA output spectrum. The correlation coefficients calculated from the measured data for both the SOA input (thermal light) and output are shown in Fig. 14, along with simulation results. The thermal-light measurements were obtained by removing the SOA from the experimental setup shown in Fig. 12. Filter B was tuned from $-0.4 \mathrm{~nm}$ to $+0.3 \mathrm{~nm}$, relative to the center wavelength of filter $\mathrm{A}$. Note that due to limitations in available filters, filter $\mathrm{A}$ is not aligned to the center of the input slice. For thermal light [Fig. 14(a)], we see that the correlation coefficient approaches zero as filter B is detuned from filter $\mathrm{A}$. The correlation observed at small detunings is due to the spectral overlap between the filters, and as expected, when the bands are disjoint, the correlation is negligible.

In comparison, Fig. 14(b) clearly shows negative intensity correlations between the different spectral components of the SOA output. These anticorrelations exist both within the original signal bandwidth $(\rho<0$ at $-0.2 \mathrm{~nm}$ offset) and between the new frequency components generated by the nonlinearities of the SOA. For filter detunings of \pm 0.1 , this effect is partially masked by the significant overlap of the filters. These results confirm the nonthermal properties of the SOA output light and substantiate our understanding of the origins of post-SOA filtering.

When the amplifier is saturated, the device gain is inversely proportional to the input intensity, and thus acts to compress
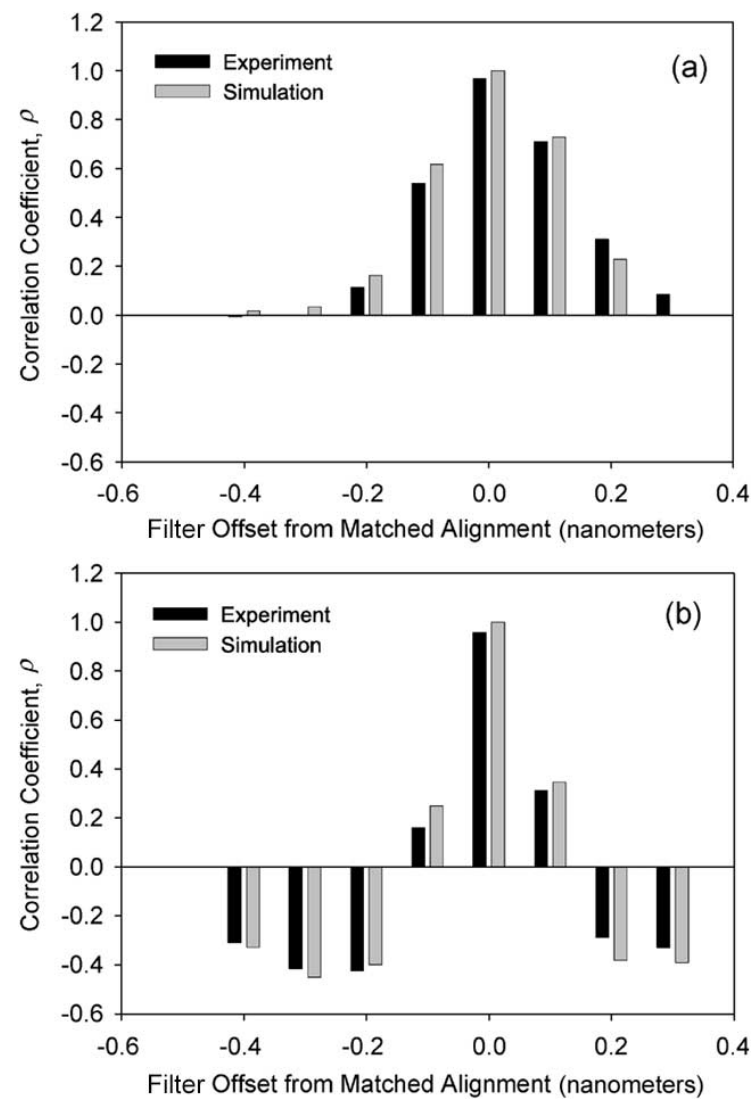

Fig. 14. Correlation coefficient $\rho$ of (a) the spectrum-sliced input light and (b) the SOA output light, as a function of filter B offset.

any intensity fluctuations present in the input light. This gain compression is approximately uniform across the entire gain bandwidth [24]. The fluctuations of an individual frequency component inversely modulate the gain across the amplifier bandwidth, and this gain modulation is, in turn, imprinted onto the other spectral components propagating through the amplifier. This results in the observed anticorrelation between the different spectral components present in the SOA output light. The superposition of these anticorrelated noise components then yields reduced fluctuations in the total output intensity. Optical filtering after the SOA removes frequency components that contribute to the total noise suppression, thus compromising the added benefit of the technique.

As the noise suppression depends on the correlation between the spectral components of the SOA output, we would further expect that a phase decorrelation of the various optical frequencies would also lead to RIN degradation. To investigate this, we measured the RIN at the SOA output for varying fiber lengths, i.e., varying levels of dispersion. Our results are shown in Fig. 15. Also shown is the system-output RIN for the spectrum slice without noise suppression, which as expected, is unaffected by dispersion. However, the RIN of the SOA output light clearly degrades with increasing dispersion. This effect becomes observable as the introduced group delay approaches the period of the suppressed noise fluctuations.

At very low levels of dispersion, the measured SOA output RIN exhibits a steeper rise than the corresponding calculated values. We also observed that adding a 5-nm ASE blocking 


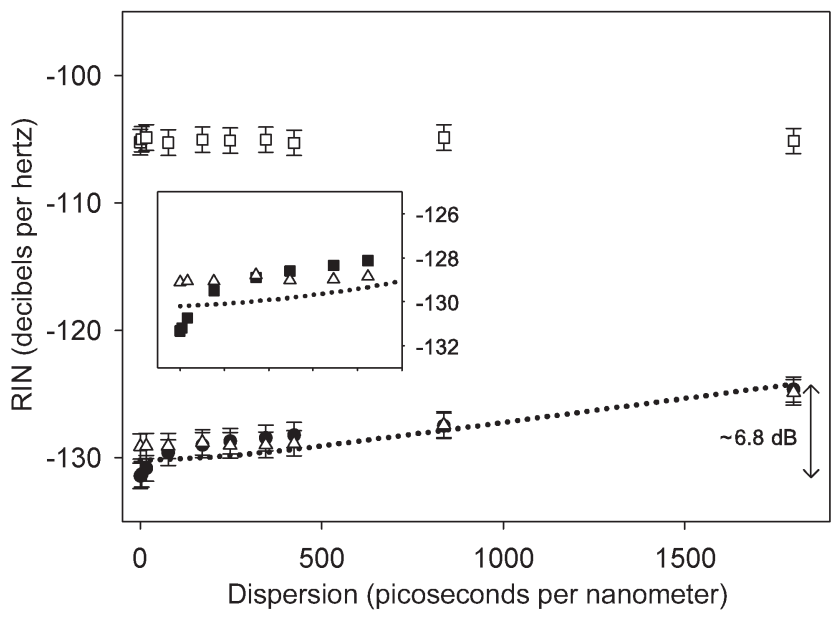

Fig. 15. RIN versus dispersion: Measured SOA input ( $\square$ ) and SOA output RIN (•), predicted SOA output RIN (...) and measured SOA output RIN with ASE blocking filter $(\triangle)$. Inset shows SOA output RIN for dispersion values ranging from 0 to $500 \mathrm{ps} / \mathrm{nm}$; caption is as above.

filter at the SOA output removes this trend. This suggests that the discrepancy is caused by the absence of amplifier noise in the simulations, supporting the previous suggestions that ASE participates in the nonlinear noise-suppression process. Due to its wide bandwidth, the ASE rapidly loses correlation with increasing dispersion. This eliminates the ASE contribution to the noise suppression, as seen from the good agreement between experiment and simulation at higher levels of dispersion. We have also confirmed that the dispersion-induced signal degradation can be overcome by employing dispersion compensation.

\section{SyStem IMPLICATIONS}

The spectral broadening of the channel bandwidth at the SOA output and the deleterious effects of onward filtering give rise to a tradeoff between intensity noise and crosstalk in a high-channel-density spectrum-sliced system. Steeper filtering at the receiver is required to minimize crosstalk, which in turn leads to a degradation in signal quality. An optimum receiver bandwidth is therefore required to maximize system performance.

We investigated this tradeoff in a $3 \times 2.5-\mathrm{Gb} / \mathrm{s}$ spectrumsliced system [25]. Both the crosstalk and the intensity noise are influenced by the receiver/demultiplexer filter bandwidth. With good receiver-filter extinction, the crosstalk will be dominated by the two adjacent channels. For this study, we assume that the three-channel performance is sufficient to represent a higherchannel-count WDM system.

A polarized ASE source was spectrally sliced into three channels using 0.24-nm filters. The center wavelength of the subject channel (channel 1) was fixed at $1551.3 \mathrm{~nm}$, while the crosstalk channels (channels 2 and 3 ) were tuned to different wavelengths, in order to achieve different channel spacings. An EDFA was used after each channel filter, in order to ensure sufficient input power to saturate the SOA. The three channels were individually modulated with NRZ data at $2.5 \mathrm{~Gb} / \mathrm{s}$ using $\mathrm{LiNbO}_{3}$ modulators. At the receiver, channel 1 was de-

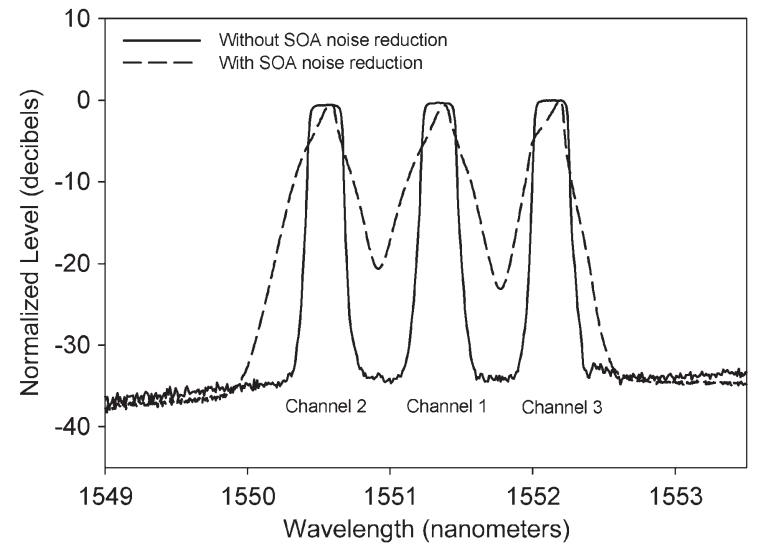

Fig. 16. Receiver input spectra with and without SOA-based noise reduction.

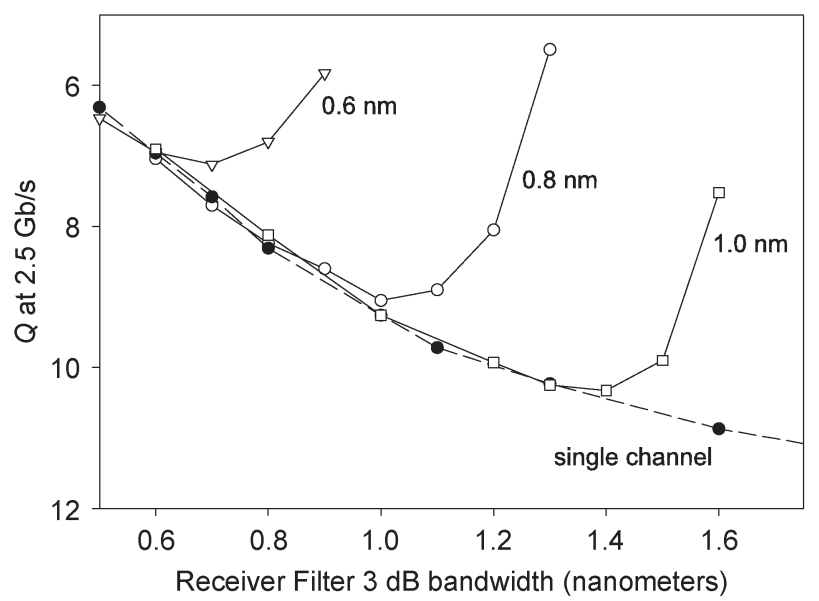

Fig. 17. Three-channel-system $Q$ measurement at $0 \mathrm{dBm}$ for varying filter widths and channel spacings of $0.6,0.8$, and $1.0 \mathrm{~nm}$ (open symbols). Singlechannel measurements are shown for comparison $(\bullet)$.

multiplexed and a high-speed sampling oscilloscope was used to detect the filtered signal and measure the system $Q$ as a function of the receiver-filter bandwidth. The receiver filter was a bandwidth-tunable FBG, with negligible dispersion at $2.5 \mathrm{~Gb} / \mathrm{s}$. Fig. 16 shows the receiver input spectrum for the $0.8-\mathrm{nm}$ $(100 \mathrm{GHz})$ channel-spacing configuration, with and without SOA-based noise reduction.

The system performance is given in Fig. 17 for channel spacings of $0.6,0.8$, and $1.0 \mathrm{~nm}$. Note that the receiver filter had good out-of-band extinction (typically $>30 \mathrm{~dB}$ ), thus minimizing the crosstalk from outside of the receiver-filter passband. As expected, the broader receiver bandwidths allow more crosstalk from the adjacent channels. The dashed baseline shown in the graph is the corresponding $Q$ measurement for the single-channel scenario, which represents the intensity-noise limit for the system.

From Fig. 17, the optimum channel bandwidth for the $0.6-, 0.8-$, and $1.0-\mathrm{nm}$ channel spacings are $0.7,1.0$, and $1.4 \mathrm{~nm}$, respectively. The system is intensity-noise limited for bandwidths less than the optimum value, after which it becomes crosstalk limited. In each case, the optimum receiver/demux filter bandwidth is greater than the channel spacing, which is in contrast to the system without SOA noise reduction, where the optimal receiver-filter bandwidth is approximately 


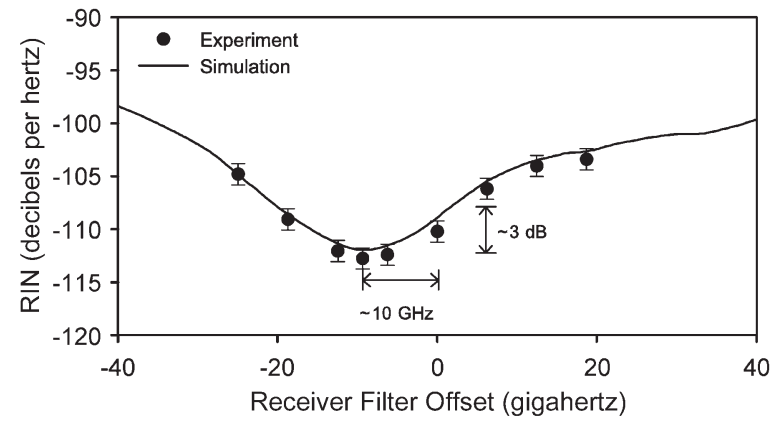

Fig. 18. RIN at receiver-filter output, as a function of frequency offset between the $0.24-\mathrm{nm}$ input spectrum slice and a $0.24-\mathrm{nm}$ receiver filter (same shape as slicing filter).

half the channel spacing [26]. In systems using SOA-based noise suppression, receiver filtering results in a more dramatic increase in intensity noise in comparison with the thermal light case. This results in the optimum receiver bandwidth being shifted toward a higher level of adjacent-channel overlap. This study clearly demonstrates the need to consider the effects of channel and receiver filter shape/width in the design of highchannel-count spectrum-sliced systems employing SOA-based noise reduction.

\section{IMPROVING POST-SOA-FILTERED SIGNAL QUALITY}

In view of the preceding results, we next discuss techniques to overcome the post-SOA filtering effects and improve the signal quality at the receiver. The following investigations use the SOA in deep saturation as outlined in Section II. Operating the SOA at lower saturation levels can reduce spectral broadening, lessening the effects of optical filtering at the receiver. However, this will also be accompanied by reduced noise suppression and increased ASE at the SOA output, yielding little, if any, overall benefit for a multichannel system with reasonable receiver-filter widths. In this section, we focus on techniques that provide improved signal quality, given that the SOA is optimized for best noise suppression.

\section{A. Red-Shift Compensation}

One simple technique that makes a noticeable improvement in signal RIN is detuning of the receiver filter to align with the shifted peak of the SOA output. As discussed previously, a red shift in the peak wavelength occurs as the light travels through the saturated amplifier. By positioning the post-SOA receiver filter to account for this frequency shift relative to the input spectrum slice, we observed a noticeable improvement in signal quality. Fig. 18 shows the RIN after receiver filtering as a function of the frequency offset between the 0.24-nm input spectrum slice and the receiver filter. The lowest RIN is obtained when the receiver filter is aligned $\sim 10 \mathrm{GHz}$ $(0.08 \mathrm{~nm})$ below the center frequency of the input spectrum slice. The observed RIN improvement is due to the decrease in filtering of the high-intensity spectral components present in the SOA output light.

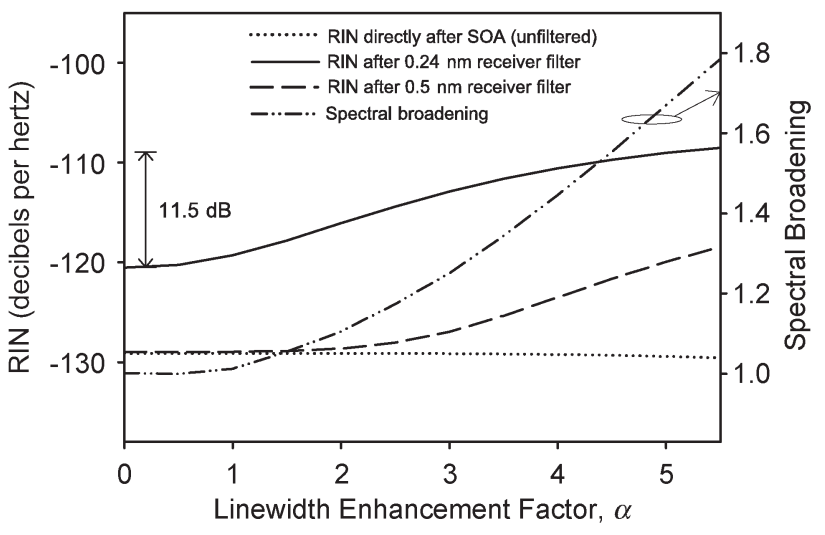

Fig. 19. Simulation results for varying $\alpha$ : Unfiltered SOA output RIN, RIN at filter output (0.24- and 0.5-nm filters), and spectral broadening (ratio of SOA output $10-\mathrm{dB}$ bandwidth to SOA input $10-\mathrm{dB}$ bandwidth).

\section{B. SOA Design Optimizations}

1) Linewidth-Enhancement Factor: We have also used the numerical model to investigate potential improvement in received signal quality by optimized SOA design. The phase modulation and FWM processes within the SOA are governed by the device linewidth-enhancement factor $\alpha$ [18], [27]. As these effects are responsible for the observed spectral distortion, we used simulations to assess the impact of $\alpha$ on the postSOA filtered signal. Using a 0.24-nm input spectrum slice, we calculate the RIN as a function of $\alpha$, directly after the SOA, and at the output of the receiver filter. Simulation results are shown in Fig. 19 for post-SOA receiver filters (aligned to input slice) of 0.24- and 0.5-nm bandwidths.

As expected [19], the noise suppression directly after the SOA (shown by the dotted curve in Fig. 19) is not affected by $\alpha$. However, $\alpha$ clearly has a strong effect on the post-SOA-filtered signal quality. Typical bulk SOAs have linewidth-enhancement factors between 3 and 8 ( $\alpha=5$ was used as a best fit to our experimental data). Reducing the linewidth-enhancement factor of semiconductor laser diodes is a subject of ongoing research, and advances in this area have shown that $\alpha$ can be reduced by optimizing the design of the device. Particularly, optimized quantum-well and quantum-dot structures have been shown to yield lower linewidth-enhancement factors [28], [29]. By reducing $\alpha$ in our simulations, we obtain a maximum RIN improvement of $11.5 \mathrm{~dB}$ over our experimental values for the 0.24-nm filter and a 9-dB improvement with the $0.5-\mathrm{nm}$ filter. The spectral broadening at the SOA output is also shown relative to the input slice bandwidth, illustrating the significant influence of the SOA linewidth-enhancement factor on spectral efficiency. As $\alpha$ is reduced, the spectral broadening due to phase-modulation-induced chirp decreases, as does the FWM. This reduces the impact of post-SOA filtering, resulting in improved RIN. For $\alpha=0$, slight broadening occurs in the tails of the spectrum [20], due to remaining FWM contributions. These simulation results clearly show that the post-SOAfiltered signal quality can be improved significantly by reducing the linewidth-enhancement factor.

2) Length: In Section II, we discussed the use of multistage SOAs for improved noise suppression. Similar benefits are possible by increasing the length of the single-stage device. 


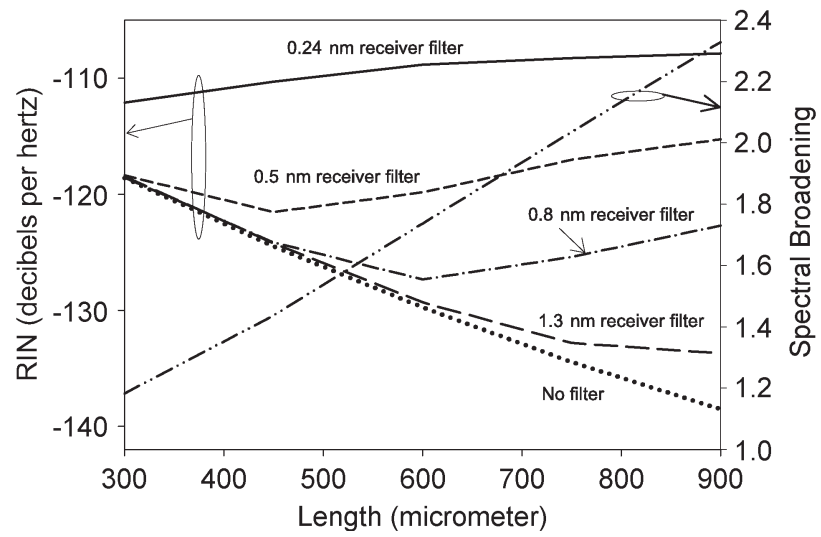

Fig. 20. Simulation results for varying device length $L$ : Unfiltered SOA output RIN (dots), RIN at filter output [1.3- (long dashes), 0.8- (dash-dots), 0.5 - (short dashes), and 0.24-nm filters (solid)], and spectral broadening (ratio of SOA output 10-dB bandwidth to SOA input 10-dB bandwidth, given by gray curve). Device current per unit length was held fixed.

We examined this using simulations, where we calculated the RIN as a function of SOA length for different receiver-filter bandwidths (Fig. 20).

Optimizations of device parameters (e.g., gain, area) to allow for superior noise suppression was also discussed briefly in [15]. However, better noise suppression at the SOA output does not necessarily result in improved post-SOA-filtered signal quality, and this should be considered when optimizing device parameters. For example, from Fig. 20, we see that as the length $L$ increases, the broadening significantly increases (shown by the dash-dot-dot line), while also providing higher noise-suppression benefit at the SOA output (dotted line). Consequently, there is an optimum device length at the point where the increased suppression benefits balance the post-filtering effects. For the 0.24-nm receiver filter, the filtering effects dominate and the received signal quality does not improve with increased device length.

Similar results are obtained in simulations when varying the device gain. Note that decreasing $\alpha$ in the design will reduce the impact of post-SOA filtering, allowing RIN improvement with increasing device length (or gain).

\section{SOA FOR NOISE SUPPRESSION AND MOdUlation}

One of the benefits of using an SOA in a spectrum-sliced system is that it can be used both as amplifier and modulator, resulting in cost savings for access applications. Previous studies [9] have demonstrated the feasibility of this approach by using a saturated SOA to modulate a $0.6-\mathrm{nm}$ spectrum slice at $600 \mathrm{Mb} / \mathrm{s}$, yielding a lower error floor than an EOM without noise suppression. However, to the authors' knowledge, the noise suppression offered by a modulated SOA has not been previously compared with the noise performance in $\mathrm{CW}$ operation.

We have characterized the received signal quality when the SOA is used for simultaneous modulation and noise reduction. This is compared with the performance of the SOA/EOM combination, in order to assess the penalty, if any, imposed on the noise-suppression performance by modulating the SOA.

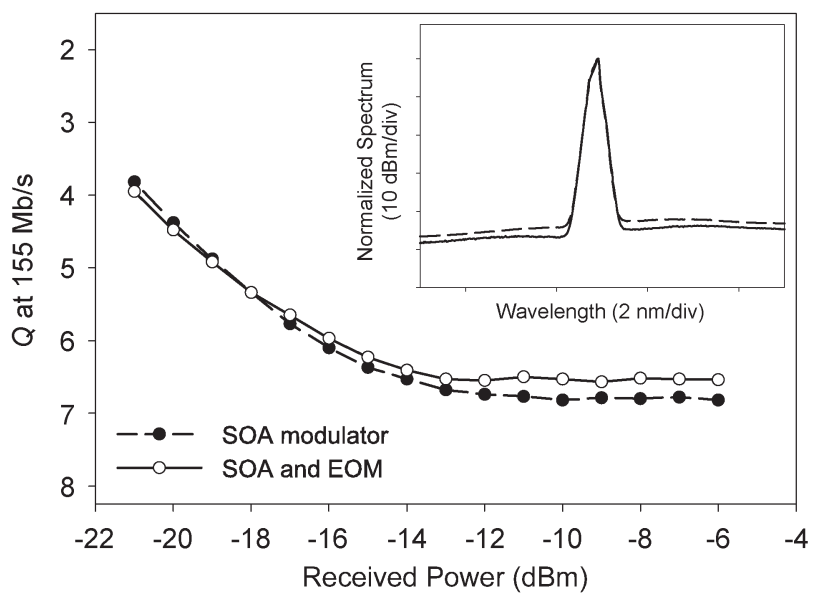

Fig. 21. System $Q$ at $155 \mathrm{Mb} / \mathrm{s}$ as a function of the received power for the saturated SOA modulator (black dash) and the saturated SOA/EOM (solid line). Detector bandwidth is $2.85 \mathrm{GHz}$.

A commercial diode driver was used to modulate the drive current of the (JDS) SOA, and $Q$ measurements were performed at $155 \mathrm{Mb} / \mathrm{s}$ using a sampling oscilloscope and a $2.85-\mathrm{GHz}$ receiver module. The 0.24-nm spectrum slice shown in Fig. 7(a) was used as the SOA input.

As seen in Fig. 21, the modulated SOA gives slightly better performance than the SOA/EOM combination. By comparison, the $Q$ for the system with no SOA is $\sim 2.5$. The slight noise disadvantage of the EOM is attributed to the polarization sensitivity of the external modulator, which degrades the noise suppression of the SOA by blocking the orthogonally polarized components of the ASE. This effect is visible in the inset of Fig. 21, where the ASE level of the EOM output is $\sim 3 \mathrm{~dB}$ lower than that of the SOA output.

However, using an optimized receiver with added electrical filtering will improve the received signal quality, giving advantage to the higher extinction ratio of the EOM (The extinction ratio of the EOM used in this experiment is $\sim 3 \mathrm{~dB}$ greater than that of the $\mathrm{SOA}$ ). The $\mathrm{LiNbO}_{3}$ modulator is also expected to provide better performance than the $\mathrm{SOA}$ at bit rates exceeding several gigahertz, due to the limited switching time of the amplifier. Nevertheless, in cost-sensitive lower-bit-rate applications such as PONs, the saturated SOA modulator is an attractive choice.

\section{CONCLUSION}

This paper presents a comprehensive study of the ramifications of incorporating a saturated semiconductor optical amplifier (SOA) at the transmitter for intensity-noise suppression of incoherent light. We have seen that the suppression benefit provided by the SOA can be compromised by spectral broadening and subsequent optical filtering. Correlation characterizations performed on the intensity-smoothed light show strong anticorrelations between the intensity fluctuations of the constituent spectral components. The observed increase in noise due to filtering originates from the loss of these intensity correlations when the SOA output spectrum is altered. Any occurrence that affects these correlations, such as dispersion or polarization, can also lead to signal degradation. 
We show that the spectral-filtering effects can be overcome by system and device-design optimizations. In particular, significant improvement in signal quality can be achieved by decreasing the device linewidth-enhancement factor $\alpha$ while maintaining a high level of gain compression. This would ensure excellent noise suppression, while minimizing the spectral broadening. It is worth noting that recent progress in the development of quantum-dot SOAs shows potential in achieving these requirements [30].

We have also used the saturated SOA as a modulator and observed no penalty to the noise-suppression process. Further cost savings can be achieved by integrating multiple channels using a single-chip SOA array [9] for downstream-link deployment. The simplicity and effectiveness of using the SOA for simultaneous noise suppression, modulation, and amplification, renders it an attractive choice for cost-sensitive spectrum slicing applications.

\section{REFERENCES}

[1] D. W. Faulkner and Y. Maeda, "PON systems standards developments in FSAN and ITU-T," in Proc. Eur. Conf. Optical Communications (ECOC), Rimini, Italy, 2003, pp. 20-23, paper Mo3.1.4.

[2] K. Akimoto, J. Kani, M. Teshima, and K. Iwatsuki, "Super-dense WDM transmission of spectrum-sliced incoherent light for wide-area access network," J. Lightw. Technol., vol. 21, no. 11, pp. 2715-2721, Nov. 2003.

[3] J. S. Lee, Y. C. Chung, and D. J. DiGiovanni, "Spectrum-sliced fiber amplifier light source for multichannel WDM applications," IEEE Photon. Technol. Lett., vol. 5, no. 12, pp. 1458-1461, Dec. 1993.

[4] G. J. Pendock and D. D. Sampson, "Transmission performance of high bit rate spectrum-sliced WDM systems," J. Lightw. Technol., vol. 14, no. 10, pp. 2141-2148, Oct. 1996.

[5] A. J. Keating and D. D. Sampson, "Reduction of excess intensity noise in spectrum-sliced incoherent light for WDM applications," J. Lightw. Technol., vol. 15, no. 1, pp. 53-61, Jan. 1997.

[6] J. H. Han, J. W. Ko, J. S. Lee, and S. Y. Shin, "0.1-nm narrow bandwidth transmission of a $2.5 \mathrm{~Gb} / \mathrm{s}$ spectrum-sliced incoherent light channel using an all-optical bandwidth expansion technique at the receiver," IEEE Photon. Technol. Lett., vol. 10, no. 10, pp. 1501-1503, Oct. 1998.

[7] F. Koyama, "High power superluminescent diodes for multi-wavelength light source," in IEEE Lasers and Electro-Optics Society (LEOS) Аnnu. Meeting, San Francisco, CA, 1999, pp. 333-334, paper TuY2.

[8] S.-J. Kim, J.-H. Han, J.-S. Lee, and C.-S. Park, "Intensity noise suppression in spectrum-sliced incoherent light communication systems using a gain saturated semiconductor optical amplifier," IEEE Photon. Technol. Lett., vol. 11, no. 8, pp. 1042-1044, Nov. 1999.

[9] F. Koyama, T. Yamatoya, and K. Iga, "Highly gain-saturated GaInAsP/InP SOA modulator for incoherent spectrum-sliced light source," in Proc. Conf. Indium Phosphide and Related Materials, Williamsburg, VA, 2000, pp. 439-442.

[10] P. Healey et al., "Spectral slicing WDM-PON using wavelength-seeded reflective SOAs," Electron. Lett., vol. 37, no. 19, pp. 1181-1182, 2001

[11] A. D. McCoy, B. C. Thomsen, M. Ibsen, and D. J. Richardson, "Experimental study on receiver filtering effects in a spectrum-sliced incoherent light WDM system using SOA based noise reduction," in Proc. Eur. Conf. Optical Communications (ECOC), Rimini, Italy, 2003, pp. 866867, paper We4P155.

[12] H. Hodara, "Statistics of thermal and laser radiation," IEEE Proc., vol. 53, no. 7, pp. 696-704, 1965.

[13] P. R. Morkel, R. I. Laming, and D. N. Payne, "Noise characteristics of high-power doped-fibre superluminescent sources," Electron. Lett., vol. 26, no. 2, pp. 96-98, 1990.

[14] H. S. Kim, R. P. H. Haaksman, T. P. Newson, and D. J. Richardson, "Noise properties and phase resolution of interferometer systems interrogated by narrowband fiber ASE sources," J. Lightw. Technol., vol. 17, no. 11, pp. 2327-2335, Nov. 1999.

[15] M. Zhao, G. Morthier, and R. Baets, "Analysis and optimization of intensity noise reduction in spectrum-sliced WDM systems using a saturated semiconductor optical amplifier," IEEE Photon. Technol. Lett., vol. 14, no. 3, pp. 390-392, Mar. 2002.

[16] M. Shtaif and G. Eisenstein, "Noise properties of nonlinear semiconductor optical amplifiers," Opt. Lett., vol. 21, no. 22, pp. 1851-1853, 1996.

[17] S.-J. Kim, J.-H. Han, J.-S. Lee, and C.-S. Park, "Suppression of intensity noise in $10 \mathrm{~Gb} / \mathrm{s}$ spectrum-sliced incoherent light channel using gain saturated semiconductor optical amplifiers," Electron. Lett., vol. 35, no. 14 , pp. 1000-1001, 1999.

[18] G. V. Agrawal and N. A. Olsson, "Self-phase modulation and spectral broadening of optical pulses in semiconductor laser amplifiers," IEEE J. Quantum Electron., vol. 25, no. 11, pp. 2297-2306, Nov. 1989.

[19] M. Munroe, J. Cooper, and M. G. Raymer, "Spectral broadening of stochastic light intensity-smoothed by a saturated semiconductor optical amplifier," IEEE J. Quantum Electron., vol. 34, no. 3, pp. 548-551, Mar. 1998.

[20] A. D. McCoy, P. Horak, B. C. Thomsen, M. Ibsen, and D. J. Richardson, "Improving signal quality in a spectrum-sliced WDM system using SOAbased noise reduction,"IEEE Photon. Technol. Lett., vol. 17, no. 1, pp. 241-243, Jan. 2005.

[21] A. Bilenca and G. Eisenstein, "Statistical noise properties of an optical pulse propagating in a nonlinear semiconductor optical amplifier," IEEE J. Quantum Electron., vol. 41, no. 1, pp. 36-44, Jan. 2005.

[22] M. Shtaif and G. Eisenstein, "Experimental study of the statistical properties of nonlinearly amplified signals in semiconductor optical amplifiers," IEEE Photon. Technol. Lett., vol. 9, no. 7, pp. 904-906, Jul. 1997.

[23] A. Papoulis, Probability, Random Variables, and Stochastic Processes, 3rd ed. New York: McGraw-Hill, 1991.

[24] L. Kazovsky and S. Benedetto, Optical Fiber Communication Systems. Norwood, MA: Artech House, 1996.

[25] A. D. McCoy, B. C. Thomsen, M. Ibsen, and D. J. Richardson, "Filtering effects in a spectrum-sliced WDM system using SOA based noise reduction," IEEE Photon. Technol. Lett., vol. 16, no. 2, pp. 680-682, Feb. 2004.

[26] K. Akimoto, J. Kani, M. Teshima, and K. Iwatsuki, "Spectrum-sliced 25-GHz spaced, 155 Mbps $\times 32$ channel WDM access," in Proc. Conf. Lasers and Electro-Optics (CLEO), Baltimore, MD, 2001, pp. II-556-II557.

[27] S. Diez, C. Schmidt, R. Ludwig, H. G. Weber, K. Obermann, S. Kindt, I. Koltchanov, and K. Petermann, "Four-wave mixing in semiconductor optical amplifiers for frequency conversion and fast optical switching," IEEE J. Quantum Electron., vol. 3, no. 5, pp. 1131-1145, May 1997.

[28] M. P. Mullane and J. G. McInerney, "Sensitivity of the linewidth enhancement factor to structure and threshold level in strained semiconductor lasers," in Proc. Conf. Lasers and Electro-Optics (CLEO), San Francisco, CA, 2000, pp. 180-181, paper CTuA60.

[29] J. Stohs, D. J. Bossert, D. J. Gallant, and S. R. J. Brueck, "Gain, refractive index change, and linewidth enhancement factor in broad-area GaAs and InGaAs quantum-well lasers," IEEE J. Quantum Electron., vol. 37, no. 11, pp. 1449-1459, Nov. 2001.

[30] T. W. Berg and J. Mørk, "Ultrafast optical signal processing using semiconductor quantum dot amplifiers," in IEEE Lasers and Electro-Optics Society (LEOS) Annu. Meeting, Glasgow, Scotland, 2002, pp. 321-322, paper TuZ2.

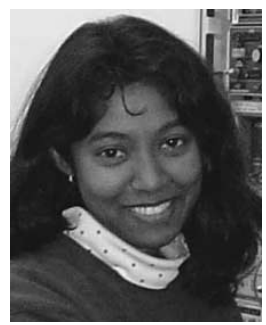

Anoma D. McCoy (S'02-M'04) received the B.S. degree in electrical engineering from Grove City College, Grove City, PA, and the M.S. degree in electrical engineering from Purdue University, West Lafayette, IN. She is currently working toward the $\mathrm{Ph} . \mathrm{D}$. degree at the Optoelectronics Research Centre, University of Southampton, U.K.

She then went on to join the test and measurement division of Hewlett-Packard (now Agilent Technologies) as a Systems Design Engineer, where shefocused on designing test systems for fiber-optic communications. Her interests include low-cost optical sources and techniques for Metro/LAN applications, source-noise reduction techniques, and semiconductor optical amplifiers and their nonlinear applications. 


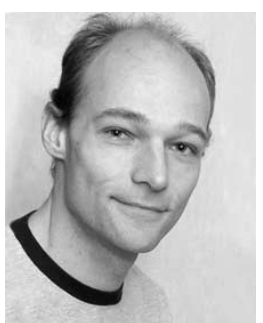

Peter Horak received the M.Sc. degree in theoretical physics, and the Ph.D. degree in theoretical quantum optics from the University of Innsbruck, Austria, in 1993 and 1998, respectively.

He joined the Optoelectronics Research Centre, University of Southampton, U.K., in 2001, where he is now a Senior Research Fellow. His current research interest is in the theory and modeling of lownoise optoelectronics devices, and their applications in classical and quantum communication systems.

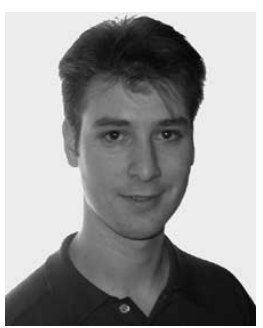

Benn C. Thomsen received the B.Tech. (optoelectronics) degree with first-class honors, the M.Sc. degree with distinction, and the Ph.D. degree in physics from The University of Auckland, New Zealand. His $\mathrm{Ph}$.D. research involved the development and characterization of short optical-pulse sources suitable for high-capacity optical communication systems and the development of nonlinear switching technologies for high-speed all-optical demultiplexing in opticaltime-division-multiplexing systems.

He then joined the Optoelectronics Research Centre, Southampton University, U.K., as a Research Fellow in 2002, where he carried out research on ultrashort optical-pulse generation and characterization, optical packet switching based on optically coded labels, and all-optical pulse processing. He joined the Optical Networks Group at University College London, London, U.K., in 2004, and his current research focuses on all-optical regeneration within dynamic optical networks. He has authored/coauthored over 50 papers in journals and conferences in the area of optical communication and optical pulse characterization.

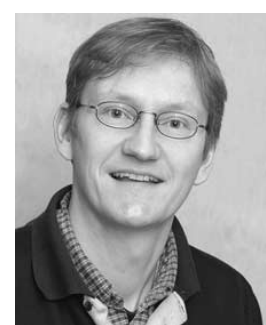

Morten Ibsen was born in Copenhagen, Denmark. He is educated in the areas of physics, mathematics, and optical communications at Institute of Physics and Astronomy (IFA), University of Aarhus, Denmark, Optical Fibre Technology Centre, University of Sydney, Australia, and Optoelectronics Research Centre, Southampton University, U.K.

$\mathrm{He}$ is currently interested in specialized Bragggrating design, fabrication, and characterization; dynamic aspects of Bragg gratings, including pulse shaping and tuneable-dispersion equalization, together with devices and systems utilizing Bragg gratings. These include single-frequency DFB fiber lasers and complex code generators in opticalcode-division multiple-access (OCDMA) applications. Furthermore, he has recently started work on the exploitation of Bragg-grating technology in medicine, therapeutic health applications, and imaging.

Dr. Ibsen is currently a holder of a Royal Society of London University Research Fellowship (URF) and is a Member of the Optical Society of America (OSA).

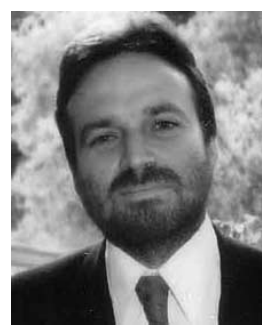

David J. Richardson was born in Southampton, England, U.K., in 1964, and received the B.Sc. and $\mathrm{Ph} . \mathrm{D}$. degrees in fundamental physics from Sussex University, U.K., in 1985 and 1989, respectively.

$\mathrm{He}$ joined the then recently formed Optoelectronics Research Centre (ORC) at Southampton University, Southampton, U.K., as a Research Fellow, in May 1989. He is now a Deputy Director of the ORC and is responsible for much of the ORC's fiberrelated activities. His current research interests include, amongst others: holey fibers, high-power fiber lasers, short-pulse lasers, optical-fiber communications, all-optical processing and switching, nonlinear optics, and the physics and applications of microstructured nonlinear/linear media. He has published more than 400 conference and journal papers in his 15 years at ORC and has produced over 15 patents. $\mathrm{He}$ is one of the founders of Southampton Photonics, Inc., a University spin-off venture that has successfully commercialized elements of high-power laser technology developed within the ORC.

Prof. Richardson was awarded a Royal Society University Fellowship in 1991, in recognition of his pioneering work on short-pulsed fiber lasers. $\mathrm{He}$ is a frequent invited speaker at leading international optics conferences in the optical communications, laser, and nonlinear optics fields, and an active member of both the national and international optics communities. 